\title{
Wormholes in Einstein-Born-Infeld Gravity
}

\author{
Jin Young Kim ${ }^{1, \star}$ and Mu-In Park ${ }^{2, \star \star}$ \\ ${ }^{1}$ Department of Physics, Kunsan National University, Kunsan 54150, Korea \\ ${ }^{2}$ Research Institute for Basic Science, Sogang University, Seoul 04107, Korea
}

\begin{abstract}
We introduce a new approach to construct wormholes without introducing exotic matters in Einstein-Born-Infeld gravity with a cosmological constant. Contary to the conventional approach, the throat is located at the place where the solutions can be joined smoothly. The metric and its derivatives are continuous so that the exotic matters are not needed there. The exoticity of the energy-momentum tensor is not essential to sustain the wormhole. We also present a method to check the stability of wormholes in the new approach.
\end{abstract}

\section{Introduction}

Wormholes are non-singular space-time structures connecting two different universes or parts of the same universe [1-3]. In the conventional approaches to construct traversable wormholes, although wormholes are exact solutions of Einstein equation, the crucial problem is the existence of exotic matter which violates energy conditions. Also the usual cuts and pastes of the throats and subsequent putting of the hypothetical matters to the throats are too artificial to be considered as a natural process.

To circumvent the obstacle by exotic matters, one may consider other extended theories of gravity so that the exotic matters are naturally generated. For example, one may consider non-minimally coupled scalar-tensor gravity theories, higher curvature gravities, higher dimensional gravities, and brane-worlds theories. A new approach for the wormhole construction has been proposed recently $[4,5]$. In the new approach, the throat is defined as the place where the solutions are smoothly joined. There, the metric and its derivatives are continuous so that the exotic matters are not introduced. In this sense the new type wormholes were named as natural wormholes to distinguish from the conventional wormholes [5].

The new approach is based on Hořava (or Hořava-Lifshitz) gravity which was proposed as a renormalizable quantum gravity based on different scaling dimensions between the space and time coordinates [6]. This new approach can be applied to other gravity theories as well when the condition for the existence of the natural throat is satisfied. Recently we have shown that this new approach is also possible in Einstein-Born-Infeld gravity with a cosmological constant [7]. In this paper, we review the geometric aspect of this new approach and introduce a method to see the stability of this new wormhole solution by computing the quasinormal modes.

\footnotetext{
$\star$ e-mail: jykim@kunsan.ac.kr

$\star \star$ e-mail: muinpark@gmail.com
} 


\section{Black hole solutions in Einstein-Born-Infeld gravity}

We consider the EBI gravity action with a cosmological constant $\Lambda$ in $D=3+1$ dimensions,

$$
S=\int d^{4} x \sqrt{-g}\left[\frac{(R-2 \Lambda)}{16 \pi G}+L(F)\right], \quad L(F)=4 \beta^{2}\left(1-\sqrt{1+\frac{F_{\mu \nu} F^{\mu \nu}}{2 \beta^{2}}}\right) .
$$

Here, the parameter $\beta$ is a coupling constant with dimensions [length $]^{-2}$ which needs to flow to infinity to recover the usual Maxwell electrodynamics with $L(F)=-F_{\mu \nu} F^{\mu \nu} \equiv-F^{2}$ at low energies. The correction terms from a finite $\beta$ represent the effect of the non-linear BI fields.

Taking $16 \pi G=1$ for simplicity, the equations of motion are

$$
\begin{gathered}
\nabla_{\mu}\left(\frac{F^{\mu v}}{\sqrt{1+\frac{F^{2}}{2 \beta^{2}}}}\right)=0, \\
R_{\mu \nu}-\frac{1}{2} R g_{\mu \nu}+\Lambda g_{\mu \nu}=\frac{1}{2} T_{\mu \nu},
\end{gathered}
$$

where the energy momentum tensor for BI fields is given by

$$
T_{\mu \nu}=g_{\mu \nu} L(F)+\frac{4 F_{\rho \mu} F_{v}^{\rho}}{\sqrt{1+\frac{F^{2}}{2 \beta^{2}}}} .
$$

Consider a static and spherically symmetric solution with the metric ansatz

$$
d s^{2}=-N^{2}(r) d t^{2}+\frac{1}{f(r)} d r^{2}+r^{2}\left(d \theta^{2}+\sin ^{2} \theta d \phi^{2}\right) .
$$

For the static electrically charged case where the only non-vanishing component of the field strength tensor is $F_{r t} \equiv E$, one can find $N^{2}(r)=f(r)$. Then, the general solution for the BI electric field is obtained from the equation (2) as

$$
E(r)=\frac{Q}{\sqrt{r^{4}+\frac{Q^{2}}{\beta^{2}}}},
$$

where $Q$ is the integration constant that represents the electric charge localized at the origin.

With (6), the Einstein equation (3) reduces to

$$
\frac{d}{d r}(r f)=1-\Lambda r^{2}+2 \beta^{2}\left(r^{2}-\sqrt{r^{4}+\frac{Q^{2}}{\beta^{2}}}\right),
$$

and a simple integration over $r$ gives

$$
f=1-\frac{2 C}{r}-\frac{\Lambda}{3} r^{2}+\frac{2 \beta^{2}}{r} \int_{0}^{r} r^{2}\left(1-\sqrt{1+\frac{Q^{2}}{\beta^{2} r^{4}}}\right) d r
$$

where $C$ is an integration constant. The solution can be expressed in terms of the hypergeometric function [8] as

$$
f(r)=1-\frac{2 M}{r}-\frac{\Lambda}{3} r^{2}+\frac{2}{3} \beta^{2} r^{2}\left(1-\sqrt{1+\frac{Q^{2}}{\beta^{2} r^{4}}}\right)+\frac{4}{3} \frac{Q^{2}}{r^{2}}{ }_{2} F_{1}\left(\frac{1}{2}, \frac{1}{4} ; \frac{5}{4} ; \frac{-Q^{2}}{\beta^{2} r^{4}}\right) .
$$


Here, $M$ is another mass parameter, defined by

$$
M \equiv C+M_{0}, \quad M_{0}=\frac{2}{3} \sqrt{\frac{\beta Q^{3}}{\pi}} \Gamma\left(\frac{1}{4}\right) \Gamma\left(\frac{5}{4}\right) .
$$

The total mass $M$, which is the sum of the intrinsic mass $C$ and the (finite) self-energy of a point charge $M_{0}$, is the usual ADM mass.

The solution (9) has a curvature singularity at the origin, but there is no curvature singularity at the horizon as expected. The solution can have two horizons generally and one can classify the black holes in terms of the values of $\beta Q$ and the cosmological constant $\Lambda$ [7].

(i) $\beta Q>1 / 2$ : In this case, the black hole may have one non-degenerate horizon (type I), or two non-degenerate (non-extremal) horizons or one degenerate (extremal) horizons (type II), depending on the mass for the asymptotically $\operatorname{AdS}(\Lambda<0)$ or flat $(\Lambda=0)$ case. There can be a phase transition from Schwarzschild (Sch)-like type I black holes to Reissner-Nodstrom(RN)-like type II black holes when the mass becomes smaller than the marginal mass $M_{0}$. When the mass is smaller than the extremal mass $M^{*}$, the singularity at $r=0$ becomes naked. In the limit $\beta \rightarrow \infty$, only the RN-like type II black holes are possible. On the other hand, for asymptotically dS $(\Lambda>0)$ case, the situation is more complicated due to the cosmological horizon. In this case, there can exist maximally three horizons (two smaller ones for black holes and the largest one for the cosmological horizon), depending on the mass and the cosmological constant.

(ii) $\beta Q=1 / 2$ : In this case, only the Sch-like type I black holes are possible. The horizon shrinks to zero size for the marginal case $M=M_{0}$, even though its mass has still a non-vanishing value. This does not mean that the metric is a flat Minkowskian. This rather means that all the gravitational energy is stored in the BI electric field as a self-energy. When the mass is smaller than the marginal mass $M_{0}$, the singularity at $r=0$ becomes naked.

(iii) $\beta Q<1 / 2$ : This case is similar to the case (ii), except that the marginal case has no (even a point) horizon so that its singularity is naked always.

\section{Construction of natural wormhole solutions}

We consider a four dimensional spherically symmetric wormhole connecting two remote parts of the same universe (or mathematically equivalently, the reflection symmetric two universes). The metric is described by

$$
d s^{2}=-N_{ \pm}(r)^{2} c^{2} d t^{2}+\frac{d r^{2}}{f_{ \pm}(r)}+r^{2}\left(d \theta^{2}+\sin ^{2} \theta d \phi^{2}\right)
$$

with two coordinate patches, each one covering the range $\left[r_{0},+\infty\right)$. In the new approach, the radius of throat $r_{0}$ is defined as

$$
\left.\frac{d N_{ \pm}}{d r}\right|_{r_{0}}=\left.\frac{d f_{ \pm}}{d r}\right|_{r_{0}}=0
$$

with the usual matching condition $N_{+}\left(r_{0}\right)=N_{-}\left(r_{0}\right), f_{+}\left(r_{0}\right)=f_{-}\left(r_{0}\right)$ by demanding that the metric and the Schwarzschild coordinates be continuous across the throat. If there exists a coordinate patch $\mathcal{M}_{+}$in which the singularities are absent for all values of $r \geq r_{0}$, one can construct a smooth regular wormhole-like geometry, which may or may not be traversable depending on the location of $r_{0}$, by joining $\mathcal{M}_{+}$and its mirror patch $\mathcal{M}_{-}$at the throat $r_{0}$. Here, it is important to note that, in the new approach, $f_{ \pm}\left(r_{0}\right)$ needs not to be vanished in contrast to the Morris-Thorne approach [2], while the quantities $d N_{ \pm}\left(r_{0}\right) / d r, d f_{ \pm}\left(r_{0}\right) / d r$ in (12) need not to be vanished in both the Morris-Thorne approach [2] and the cuts and pastes approach of Visser [3]. 
The first step to construct a natural wormhole is to find the throat radius $r_{0}$ defined by (12) with the matching condition $[4,5]$. For the case (i) of $\beta Q>1 / 2$, there is no throat for the Sch-like type I black holes with $M>M_{0}$ down to the marginal case of $M=M_{0}$, where a point-like throat is generated at the origin. The zero-size $\left(r_{0}=0\right)$ throat can grow as the black hole loses its mass. But, down to the extremal case of $M=M^{*}\left(<M_{0}\right)$, the throat is inside the outer horizon and is non-traversable. As the black hole loses its mass further, there emerges and grows a traversable throat from the extremal black hole horizon. For the AdS case, the throat grows and persists until its mass $M$ vanishes. However, for the cases of flat and dS, the throat does not grow to an indefinitely large size. There is a maximum size at a certain mass $\widetilde{M}$ before $\mathrm{M}$ vanishes, i.e., $M^{*}>\widetilde{M}>0$. If we further reduce the mass below $\widetilde{M}$, only the space-time with a naked singularity is a possible solution. So, the usual naked singularity solution for $M<M^{*}$ may be replaced by the non-singular wormhole geometry until a certain (nonnegative) mass $\widetilde{M}$ is reached in flat and dS cases. This may suggest the generalized cosmic censorship $[4,5]$ by the existence of a wormhole-like structure around the origin, which has been thought to be a singular point of black hole solutions where all the usual physical laws could break down and might be regarded as an indication of the incompleteness of general relativity.

For the case (ii) of $\beta Q=1 / 2$, a point-like throat can be generated at the origin contrary to the generation of a finite-size throat for the case (i). But its growing process is the same as the case (i), showing the existence of a maximum size of the throat with the mass $\widetilde{M}>0$ for flat and dS cases, and $\widetilde{M}=0$ for AdS case.

For the case (iii) of $\beta Q<1 / 2$, the natural wormhole geometry exists only for AdS case and its growing behavior is similar to the case (ii). On the other hand, for the flat and dS cases, only the Sch-like type I black hole solution for $M>M_{0}$ or the naked singularity solution for $0<M<M_{0}$ can exist.

Here, we have considered only the geometric aspects in constructing the natural wormhole. The analysis on the Born-Infeld field shows that the Born-Infeld eauation is not modified [7].

\section{Stability of natural wormhole solutions}

One easy way to see the stability of black hole or wormhole is to study the scalar perturbation. We consider the minimally coupled scalar wave equation

$$
\nabla^{2} \Phi=m^{2} \Phi
$$

where $m$ is mass of the scalar field. The mode solution can be studied with the following form

$$
\Phi(t, r, \theta, \phi)=\frac{\psi(r)}{r} Y(\theta, \phi) e^{-i \omega t},
$$

where $Y(\theta, \phi)$ is the spherical harmonics on $S^{2}$. In general. it is not easy to solve the equation analytically. The stability of this new type of wormhole can be considered in analogy with the quasinormal modes in black hole physics. There are several ways to compute quasinormal modes in the literature. Here, we follow the technique developed by Horowitz and Hubeny [9].

Since we are interested in the mode solution of the form $e^{-i \omega\left(t+r_{*}\right)}$, with a new radial variable $d r_{*}=d r / f(r)$, near the throat, it is convenient to consider the ingoing Eddington coordinates, $v=t+r_{*}$,

$$
d s^{2}=-f d v^{2}+2 d v d r+r^{2} d \Omega_{2}^{2} .
$$

Then the scalar wave equation (13) reduces to the following equation for the radial equation $\psi(r)$

$$
f(r) \frac{d^{2}}{d r^{2}} \psi(r)+\left(f^{\prime}-2 i \omega\right) \frac{d}{d r} \psi(r)-V(r) \psi(r)=0, \quad V(r)=\frac{f^{\prime}}{r}+\frac{\ell(\ell+1)}{r^{2}}+m^{2},
$$


where $\ell(\ell+1)$ is the laplacian on $S^{2}$ and prime denotes the derivative with respect to $r$.

The stability can be studied by finding the complex $\omega$ such that equation (16) has a solution with only ingoing modes near the throat and vanishing at infinity. If we introduce a new variable $x \equiv 1 / r$, equation (16) becomes

$$
s(x) \frac{d^{2} \psi}{d x^{2}}+\frac{t(x)}{x-x_{0}} \frac{d \psi}{d x}+\frac{u(x)}{\left(x-x_{0}\right)^{2}} \psi=0,
$$

with

$$
s(x)=-\frac{x^{4} f(x)}{x-x_{0}}, \quad t(x)=-2 x^{3} f-x^{4} \dot{f}-2 i \omega x^{2}, \quad u(x)=\left(x-x_{0}\right)\left[\ell(\ell+1) x^{2}-x^{3} \dot{f}+m^{2},\right.
$$

where $\dot{f}=d f / d x$. One can expand $s, t$, and $u$ in powers of $\left(x-x_{0}\right)$ about the throat $x_{0}$. For example, $s(x)=\sum_{n=-1}^{\infty} s_{n}\left(x-x_{0}\right)^{n}$, where $s_{-1}=-x_{0}^{4} f_{0}, s_{0}=-4 x_{0}^{3} f_{0}, s_{1}=-(1 / 2)\left(12 x_{0}^{2} f_{0}+x_{0}^{4} \ddot{f}_{0}\right), \cdots$.

To see the leading behavior of the solution near the throat, we set $\psi=\left(x-x_{0}\right)^{\alpha}$ and put into equation (17). To the leading order, we have $\alpha(\alpha-1)=0$, which have two solutions $\alpha=0$ and $\alpha=1$. Since $\alpha=0$ corresponds to the ingoing mode, we consider a solution of the form

$$
\psi(x)=\sum_{n=0}^{\infty} a_{n}\left(x-x_{0}\right)^{n} .
$$

Substituting equation (18) into (17) and equating the coefficients of $\left(x-x_{0}\right)$ for each $n$ to zero, we have the recursion relation

$$
a_{n}=-\frac{1}{n(n-1) s_{-1}} \sum_{k=0}^{n-1}\left[k(k-1) s_{n-k-1}+k t_{n-k-1}+u_{n-k-1}\right] a_{k} .
$$

One can find the quasinormal modes from the boundary condition $\psi \rightarrow 0$ as $x \rightarrow 0(r \rightarrow \infty)$. This means that we solve $\sum_{n=0}^{\infty} a_{n}\left(-x_{0}\right)^{n}=0$ in the complex $\omega$ plane. This can be done by truncating the series and computing the partial sum as a function of $\omega$. Then one can discuss the stability of wormhole from the sign of the imaginary part of $\omega$.

\section{Acknowledgments}

MIP was supported by Basic Science Research Program through the National Research Foundation of Korea (NRF) funded by the Ministry of Education, Science and Technology (2016R1A2B401304).

\section{References}

[1] J. A. Wheeler, Ann. Phys. (N.Y.) 2, 604 (1957).

[2] M. S. Morris and K. S. Thorne, Am. J. Phys. 56, 395 (1988).

[3] M. Visser, Nucl. Phys. B 328, 203 (1989); Lorenzian wormholes (AIP Press, 1995).

[4] M. B. Cantcheff, N. E. Grandi, and M. Sturla, Phys. Rev. D 82, 124034 (2010).

[5] S. W. Kim and M.-I. Park, Phys. Lett. B 751 , 220 (2015).

[6] P. Hořava, Phys. Rev. D 79, 084008 (2009).

[7] J. Y. Kim and M.-I. Park, Eur. Phys. J. C, 76, 621 (2016).

[8] T. K. Dey, Phys. Lett. B 595, 484 (2004).

[9] G. T. Horowitz and V. E. Hubeny, Phys. Rev. D 62, 024027 (2000). 